\title{
Radial artery access for bilateral ischemic embolic stroke
}

\author{
Vipulkumar V Patel ${ }^{1}$, Robert Dana Tomalty ${ }^{2}$ and Charles A Ritchie ${ }^{1 *}$ \\ ${ }^{1}$ Mayo Clinic, Jacksonville 4500 San Pablo Rd S, Jacksonville, FL 32224, USA \\ ${ }^{2}$ Radiology of Huntsville, 2006 Franklin st SE \#200, Huntsville AL, 35801, USA
}

\begin{abstract}
Acute ischemic stroke is a very common cause of disability and death in developed countries. Intravascular thrombolysis and mechanical thrombectomy are effective mode of care for patient with acute ischemic stroke due to large vessels occlusion. Mechanical thrombectomy in a case of an acute ischemic stroke lead to favorable functional outcomes when done early. A 58-year-old man was seen in consultation after 24 hours of vascular surgery for acute aortic syndrome. The patient developed left side hemiparesis from embolic stroke after surgical intervention. We choose the radial artery access to approach the cerebral vessels for mechanical thrombectomy because of compromised femoral artery due to surgical intervention which is different from the traditional femoral artery approach. Patient had a full neurologic recovery within 12 hours after the mechanical thrombectomy.
\end{abstract}

Abbreviations: NIHHS: National Institute of Health Stroke Scale; ACA: Anterior cerebral artery; MCA: Middle cerebral artery; TICI: Thrombolysis in cerebral infarction; tPA: Tissue plasminogen activator

\section{Introduction}

Stroke is a frequent presentation in emergency departments, and it is the fifth leading cause of death in the United States and is a major cause of serious disability for adults [1]. About 795,000 people in the United States have a stroke each year [1]. The country's highest death rates from stroke are in the southeastern Unites States [1]. This case involved a 58-year-old man experiencing severe neurological deficit post operatively due to bilateral acute ischemic stroke develop from embolic occlusion of right middle cerebral artery and left internal carotid artery. Following mechanical thrombectomy patients National Institute of Health Stroke Scale (NIHSS) and local deficit improved within 12 hours of post treatment. Acute ischemic stroke is associated with significant morbidity and mortality; up to $50-80 \%$ of survivors has neurological deficit, most commonly hemiparesis, but also longterm neuropsychiatric and behavior issues. We report a case in which mechanical thrombectomy was done for the successful treatment of acute ischemic embolic stroke in a post-operative period of acute aortic syndrome and approached by right radial artery route without any complication.

\section{Case report}

A 58-year-old man was seen in consultation 24 hours after vascular surgery, bilateral open femoral artery cut down with embolectomy for acute aortic syndrome (Figure 1).The patient was placed on Clopidogrel (Bristol-Myers Squibb Co, New York, NY, USA) post operatively, no heparin. The next morning, the patient developed left sided hemiplegia and subsequently becomes obtunded requiring intubation. NIHSS was 18 prior to intubation. Non contrast computed tomography of head showed no any signs of hemorrhage. Worsening clinical presentation necessitated cerebral angiography and possible thrombectomy.

Because of the bilateral femoral cut down procedure, the right radial arterial access was used to place a 6Fr Neuro max (Pneumbra,

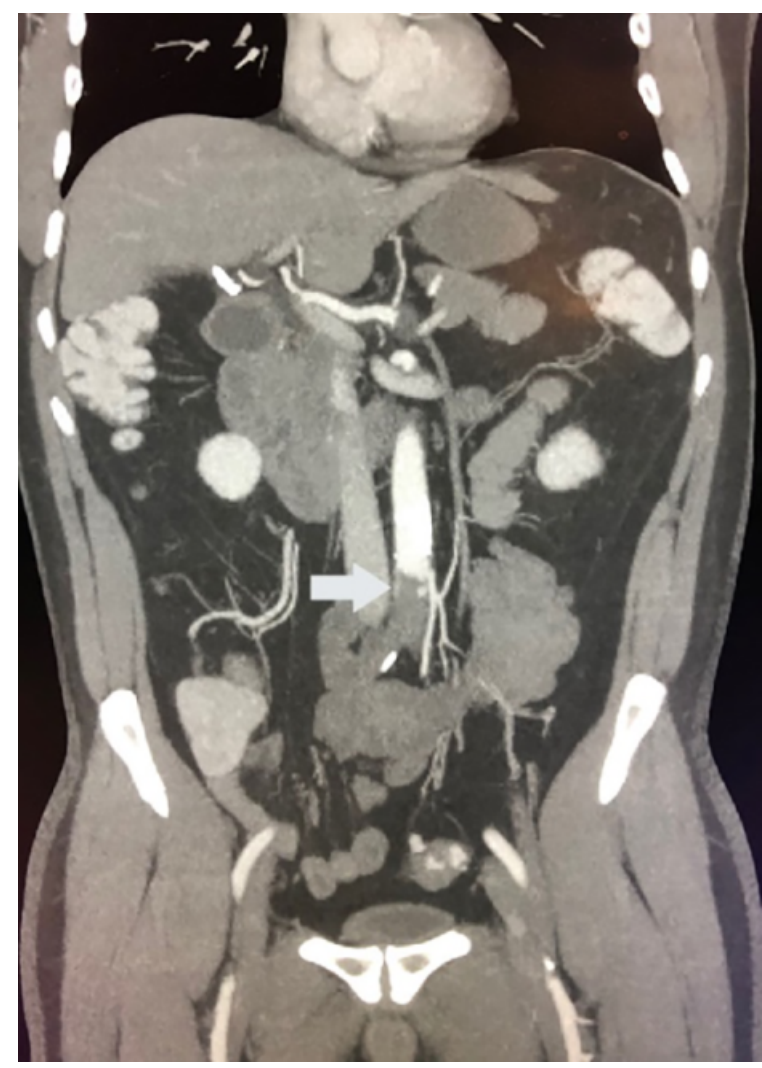

Figure 1. Contrast-enhanced coronal abdomen image demonstrates a large acute thrombus in descending aorta (arrow)

*Correspondence to: Charles Ritchie, Department of Radiology, Mayo Clinic | 4500 San Pablo Rd S| Jacksonville, FL 32224, USA, Tel. 904-953-1496; E-mail: Ritchie.charles@mayo.edu

Key words: acute ischemic stroke, radial artery access, mechanical thrombectomy Received: March 30, 2020; Accepted: April 08, 2020; Published: April 13, 2020 
Inc. Alameda, California, USA) sheath for the assumed right carotid distribution embolism.AS2 selection catheter and Jet 7 aspiration catheter (Pneumbra, Inc) where used to select the distal right internal carotid artery. Initial diagnostic angiography demonstrates a right middle cerebral artery $1^{\text {st }}$ order (M1) clot and cross filling into left anterior cerebral artery (ACA) demonstrating a filling defect in the left ACA/middle cerebral artery (MCA) (Figure 2A). A Velocity micro catheter (Pneumbra, Inc.) and Aristotle 0.14 wire (Scientia Vascular, UT, USA) were used to access the right M2 vessels. A Solitaire $6 \mathrm{~mm}$ $\mathrm{x} 40 \mathrm{~mm}$ (Medtronic, Inc. Dublin, Ireland) was deployed in the clot and retracted. After 4 minutes of removing the emboli and resulting in a thrombolysis in cerebral infarction (TICI) grade 3 on follow up angiogram (Figure $2 \mathrm{~B}$ ). The catheter system was then manipulated into the left internal carotid artery (figure 2C) and a single pass with the Solitaire $6 \mathrm{~mm} \times 40 \mathrm{~mm} / \mathrm{Jet} 7$ restored flow on left side, TICI grade 3 (figure 2D).

The patient tolerates the procedure well and after removal of clot burden from right MCA and left carotid artery terminus (Figure 3), he had a full neurologic recovery within 12 hours.

\section{Discussion}

There is a narrow window period in which restoration of cerebral blood flow is achieved by using reperfusion therapy to salvage the
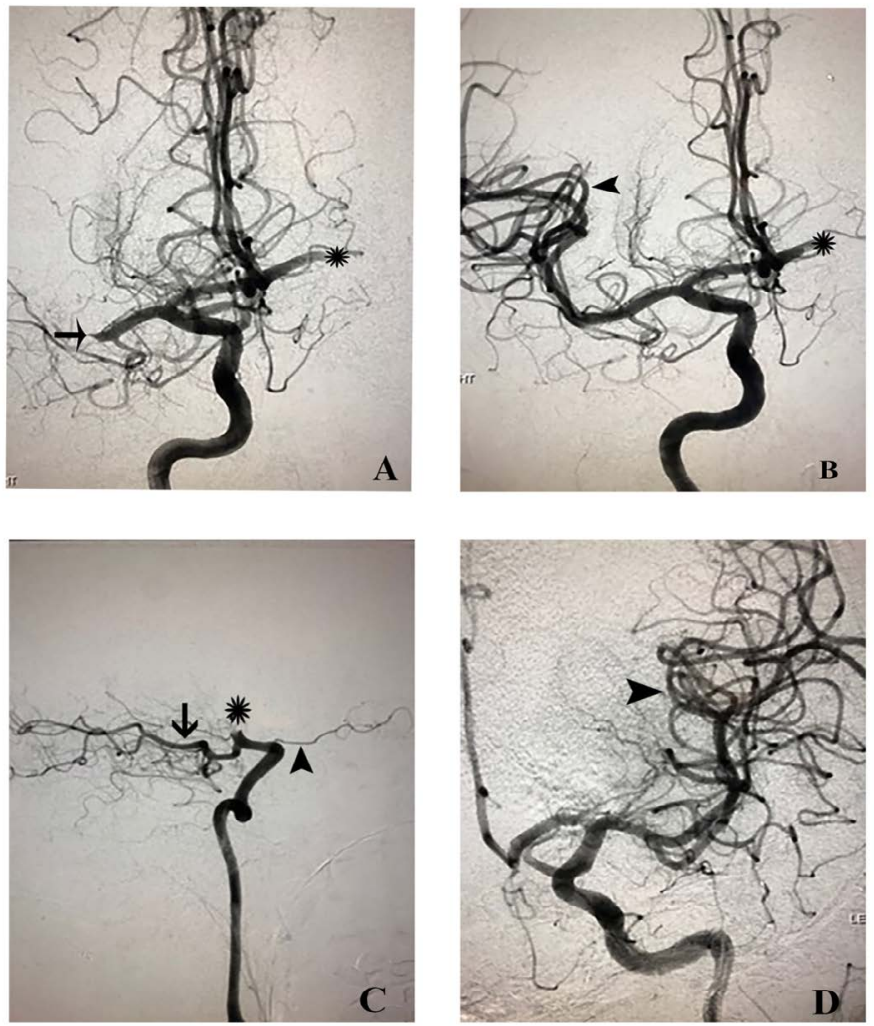

Figure 2. Demonstrates pre and post procedure angiogram of cerebral vessels. (A) Initial antero-posterior right internal carotid artery angiogram demonstrating complete occlusion of right MCA (arrow) with retrograde filling defect of the left ACA/MCA (asterisk) concerning of an additional thrombus. (B) Post treatment right internal carotid artery angiogram demonstrating complete reperfusion of the right MCA territories-TICI grade 3 (arrowhead) and continued retrograde filling defect in left ACA/MCA (asterisk). (C) Lateral projection of left internal carotid artery angiogram pre- intervention shows complete carotid terminus occlusion (T lesion) (asterisk) and no filling of left ACA/ MCA territories with a large posterior communicating artery (arrow) and left ophthalmic artery present (arrowhead). (D) Post treatment left internal carotid artery antero-posterior cerebral angiogram shows complete reperfusion in left carotid artery branches- TICI grade 3 (arrowhead)

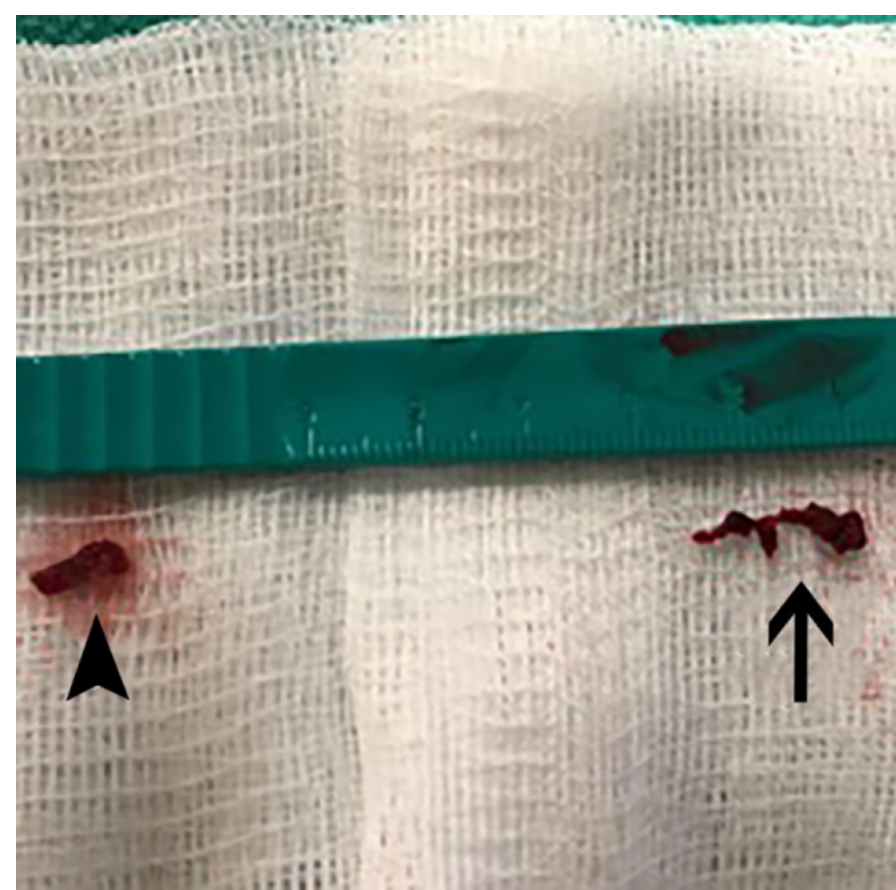

Figure 3. Post thrombectomy clot burden from the right middle cerebral artery (arrowhead) and left carotid artery terminus (arrow)

ischemic brain tissue but not yet infarcted. Most important factor in successful reperfusion therapy of acute ischemic stroke is early intervention. Majority of stroke is ischemic in origin $80 \%$ versus $20 \%$ due to brain hemorrhage. Ischemic causes are classified into intrinsic to vessels such as atherosclerosis, inflammation of vessel wall, dissection and embolism that may originate a part and travel to brain vessels or it may be the consequence of persistent hypotension because of condition like hypovolemic, cardiogenic and septic shock. However, treatment depends on the underlying etiology as well as time spent from clearly defined symptoms onset. Alteplase (recombinant tissue plasminogen activator or $\mathrm{tPA}$ ) is the mainstay of treatment of acute ischemic stroke, provided that treatment is initiated within 4.5 hours of clearly defined symptoms onset. Mechanical thrombectomy is indicated for patient with acute ischemic stroke due to large artery occlusion in the anterior circulation who can be treated within 24 hours of the times last known to be well at neurological baseline. Patient should receive intravenous Alteplase without delay, if eligible, even if considered for mechanical thrombectomy [2].

Several studies are conducted to show the importance of endovascular treatment in anterior circulation acute ischemic stroke. MR CLEAN, EXTEND-IA, and SWIFT PRIME proved the value of intrarterial thrombectomy in anterior circulation acute ischemic stroke within the first 6 hours of symptoms onset [3-5]. MR CLEAN, randomized trial of 500 patients, eligible patients are randomly assigned intraarterial treatment plus usual care or usual care alone. Arterial occlusion of anterior circulation is confirmed on vessel imaging and that could be treated within 6 hours of symptoms onset. They found an absolute difference of 13.5 percentage point in the rate of functional independence (modified Rankin score, 0 to 2 ) in favor of intervention (32.6\% vs. 19.1\%). EXTEND-IA clinical trial, randomly assigned patient with ischemic stroke with standard thrombolysis tPA treatment versus endovascular thrombectomy with solitare flow restoration stent retriever. Endovascular therapy, initiated at 210 minutes after the stroke onset, increased early neurological improvement at 3 days $(80 \%$ 
vs $37 \%$; $\mathrm{p}=0.002$ ) and improved functional outcome at 90 days, with more patient achieving functional independence (modified Rankin score 0 to 2, 71\% vs $40 \%$; $\mathrm{P}=0.01$ ). SWIFT PRIME randomized clinical trial also concluded that thrombectomy with a stent retriever within 6 hours of symptoms onset improved functional outcome at 90 days. THRACE provided additional data for thrombectomy up to 5 hours (53\% of patient with thrombectomy achieved a good clinical outcome vs $42 \%$ with medical management, $\mathrm{OR}=1.55$ ) [6]. Two another trials REVASCAT and ESCAPE provide evidence of extended time of 8 and 12 hours, respectively for mechanical thrombectomy in anterior circulation acute ischemic stroke with good clinical outcomes with mechanical thrombectomy $[7,8]$. Two recent multicenter randomized controlled trials DEFUSE 3 and DAWN suggest a late time window of 16 hours and 24 hours, respectively with a favorable outcome with mechanical thrombectomy $[9,10]$.

Imaging criteria varies according to the time of symptoms onset to determine the appropriate candidacy of patient. In above mentioned study they use different imaging criteria depending on the time lapse after onset of symptoms. Stroke severity assessed by the NIHSS to determine the eligibility for thrombectomy. MR CLEAN trials has lowest NIHSS score of 2, ESCAPE and EXTEND-IA set no specific score threshold. However, other trials have variable ranges of NIHSS score from 6 to 10. The benefits of mechanical thrombectomy in posterior circulation stroke is uncertain, but it may a reasonable treatment option for patient with posterior circulation occlusion when performed at center with appropriate expertize. Weber et al conducted an analysis on prospective multicenter revascularization in ischemic stroke patient registry and concluded that mechanical thrombectomy in posterior circulation shows a lower risk of symptomatic intracranial hemorrhage and similar effectiveness compared to anterior circulation stroke [11].

\section{Conclusion}

Our exclusive case report provides support for acute mechanical thrombectomy as a safe, effective and minimal invasive approach for occlusive acute ischemic stroke performed via radial artery access.

\section{Disclosure}

The authors whose names are listed above certify that they have NO affiliations with or involvement in any organization or entity with any financial interest (such as honoraria; educational grants; participation in speakers' bureaus; stock ownership, or other equity interest; and expert testimony or patent-licensing arrangements), or non-financial interest (such as personal or professional relationships, affiliations, knowledge or beliefs) in the subject matter or materials discussed in this manuscript.

\section{References}

1. Mozaffarian D, Benjamin E, Go A, Arnett D, Blaha M, et al. (2016) Writing Group Members; American Heart Association Statistics Committee; Stroke Statistics Subcommittee. Executive summary: heart disease and stroke statistics—2016 update: a report from the American Heart Association. Circulation 133: 447-454.

2. Powers WJ, Rabinstein AA, Ackerson T, Adeoye OM, Bambakidis NC, et al. (2018) 2018 Guidelines for the Early Management of Patients With Acute Ischemic Stroke: A Guideline for Healthcare Professionals From the American Heart Association/ American Stroke Association. Stroke 49: e46-e110. [Crossref]

3. Berkhemer OA, Fransen PS, Beumer D, van den Berg LA, Lingsma HF, et al. (2015) A randomized trial of intraarterial treatment for acute ischemic stroke. $N$ Engl $J$ Med 372: 11-20. [Crossref]

4. Campbell BC, Mitchell PJ, Kleinig TJ, Dewey HM, Churilov L, et al. (2015) Endovascular therapy for ischemic stroke with perfusion-imaging selection. $N$ Engl $J$ Med 372: 1009-18. [Crossref]

5. Saver JL, Goyal M, Bonafe A, Diener HC, Levy EI, et al. (2015) Stent-retriever thrombectomy after intravenous t-PA vs. t-PA alone in stroke. $N$ Engl J Med 372: 2285-95. [Crossref]

6. Bracard S, Ducrocq X, Mas JL, Soudant M, Oppenheim C, et al. (2016) Mechanical thrombectomy after intravenous alteplase versus alteplase alone after stroke (THRACE): a randomised controlled trial. Lancet Neurol 15: 1138-47. [Crossref]

7. Jovin TG, Chamorro A, Cobo E, de Miquel MA, Molina CA, et al. (2015) Thrombectomy within 8 hours after symptom onset in ischemic stroke. $N$ Engl J Med 372: 2296-306. [Crossref]

8. Goyal M, Demchuk AM, Menon BK, Eesa M, Rempel JL, et al. (2015) Randomized assessment of rapid endovascular treatment of ischemic stroke. N Engl J Med 372: 1019-30. [Crossref]

9. Albers GW, Marks MP, Kemp S, Christensen S, Tsai JP, et al. (2018) Thrombectomy for Stroke at 6 to 16 Hours with Selection by Perfusion Imaging. $N$ Engl J Med 378: 708-718. [Crossref]

10. Nogueira RG, Jadhav AP, Haussen DC, Bonafe A, Budzik RF, et al. (2018) Thrombectomy 6 to 24 Hours after Stroke with a Mismatch between Deficit and Infarct. N Engl J Med 378: 11-21. [Crossref]

11. Weber R, Minnerup J, Nordmeyer H, Eyding J, Krogias C, et al. (2019) Thrombectomy in posterior circulation stroke: differences in procedures and outcome compared to anterior circulation stroke in the prospective multicentre REVASK registry. Eur $J$ Neurol 26: 299-305. [Crossref]

Copyright: (C2020 Patel VV. This is an open-access article distributed under the terms of the Creative Commons Attribution License, which permits unrestricted use, distribution, and reproduction in any medium, provided the original author and source are credited. 